PROCEEDINGS OF THE

AMERICAN MATHEMATICAL SOCIETY

Volume 126, Number 10, October 1998, Pages 2987-2992

S $0002-9939(98) 04401-3$

\title{
SINGULAR EXTENSIONS OF THE TRACE AND THE RELATIVE DIXMIER PROPERTY IN THE TYPE II 1 FACTORS
}

\author{
FLORIN POP
}

(Communicated by Palle E. T. Jorgensen)

\begin{abstract}
If $N \subset M$ is an inclusion of type $\mathrm{II}_{1}$ factors with $N^{\prime} \cap M=\mathbf{C} I$, we study the connection between the existence of singular states on $M$ which extend the trace on $N$ and the Dixmier approximation property in $M$ with unitaries in $N$. We also prove the existence of singular conditional expectations from certain free product factors onto irreducible hyperfinite subfactors.
\end{abstract}

\section{INTRODUCTION}

One of the fundamental approximation properties in von Neumann algebras is the Dixmier property [4], 8.3.6. In the type $\mathrm{II}_{1}$ factorial case it states that for every element $m$ in a $\mathrm{II}_{1}$ factor $M$ and for every $\epsilon>0$, there are unitary operators $u_{1}, \ldots, u_{n}$ in $M$ and positive numbers $\lambda_{1}, \ldots, \lambda_{n}$ of sum 1 such that

$$
\left\|\sum_{i=1}^{n} \lambda_{i} u_{i}^{*} m u_{i}-\tau(m) I\right\|<\epsilon .
$$

In other words, $\tau(m) I$ belongs to the norm-closed convex hull of $\left\{u_{i}^{*} m u_{i}\right\}$. (It turns out that $\tau(m) I$ is the only element in the center of $M$ with this property.)

A natural question that arises is: if $N \subset M$ is a subalgebra, is (1) still true under the additional requirement that the unitaries $u_{i}$ belong to $N$ ? If the answer is yes, then $N$ is called a Dixmier subalgebra of $M$. The case we will be interested in is when $N$ is an irreducible subfactor, that is, a subfactor with trivial relative commutant. The relative Dixmier property has been studied by several authors (see [1] and the references therein), in connection with extensions of pure states, a problem originating in [3].

The only positive result in this direction has been recently obtained by Popa [7]: the Dixmier property (1) remains true for ultrapower factors $M^{\omega}$ and unitaries $u_{i}$ in $N^{\omega}$, where $N \subset M$ is an irreducible inclusion of (strongly) separable $\mathrm{II}_{1}$ factors.

While no example of a proper, separable Dixmier subfactor is known, in this note we construct examples of irreducible subfactors without the relative Dixmier property. We do this by studying singular states on a factor $M$ which extend the trace on a subfactor $N$. More precisely, we construct singular states on free product factors $M * N$ which extend the trace on $M$. As a consequence, $M$ is not a Dixmier

Received by the editors February 24, 1997 and, in revised form, March 10, 1997.

1991 Mathematics Subject Classification. Primary 46L10, 46L30.

(C)1998 American Mathematical Society 
subfactor of $M * N$. In particular when $M=R$, the hyperfinite $\mathrm{II}_{1}$ factor, we obtain singular conditional expectations from $R * N$ onto $R$.

Singular functionals and, more generally, singular maps between operator algebras haven't been used extensively and the existing literature on the subject is rather scarce. We refer the reader to [4], ch.10, for a presentation of the main results.

It should be noted that if in the relative Dixmier property we replace the norm by the Hilbert-Schmidt norm $\|x\|_{2}=\tau\left(x^{*} x\right)^{1 / 2}$ where $\tau$ is the trace, the situation is quite different: if $N$ is an irreducible subfactor of $M$, then for every $m$ in $M$ and for every $\epsilon>0$, there are unitary operators $u_{1}, \ldots, u_{n}$ in $N$ and positive numbers $\lambda_{1}, \ldots, \lambda_{n}$ of sum 1 such that

$$
\left\|\sum_{i=1}^{n} \lambda_{i} u_{i}^{*} m u_{i}-\tau(m) I\right\|_{2}<\epsilon .
$$

This means that $\tau(m) I$ is ultraweakly (also weakly and strongly) adherent to the convex hull of $\left\{u_{i}^{*} m u_{i}\right\}$. Property (2) is a consequence of Popa's noncommutative local Rohlin theorem [5]: with above notation, there are mutually orthogonal projections $e_{1}, \ldots, e_{n}$ in $N$ of sum 1 such that

$$
\left\|\sum_{i=1}^{n} e_{i} m e_{i}-\tau(m) I\right\|_{2}<\epsilon .
$$

Then (2) follows easily from (3) as in [2], 2.2.

\section{Singular MAPS}

In this section we discuss some properties of singular maps and functionals. We will adopt as the definition of singularity an equivalent property, due to Takesaki [8]. We refer the reader to [4], ch.10, for more information.

2.1. Definition. Let $M$ and $N$ be von Neumann algebras. A positive linear map $\phi: M \rightarrow N$ is singular if for every nonzero projection $f \in M$ there exists a nonzero projection $e \leq f$ in $M$ such that $\phi(e)=0$. If $N=\mathbf{C}$, then $\phi$ is called a singular positive functional.

2.2. Remarks. (i) If $M$ is of countable type (in particular, if $M$ is a factor) then $\phi$ is singular iff there exists an increasing sequence of projections $e_{n} \nearrow I$ such that $\phi\left(e_{n}\right)=0$.

(ii) If $\left(\phi_{n}\right)$ is a sequence of singular maps, then under any nonzero projection $f \in M$ there is a nonzero projection $e$ such that $\phi_{n}(e)=0$ for all $n$. In particular, finite sums of singular maps and point limits of sequences of singular maps are again singular.

For the following result we refer to [4], 10.1.16, for an alternate proof. We present here a different proof, more in the spirit of our approach.

2.3. Lemma. If $\phi$ is a singular positive functional on $M$ then for every nonzero $m \in M, \psi(x)=\phi\left(m^{*} x m\right)$ is singular or zero.

Proof. Write $m$ as a linear combination of four unitaries, $m=\sum_{i=1}^{4} \lambda_{i} u_{i}$. We use the Cauchy-Schwarz inequality to obtain

$$
|\psi(x)|=\left|\phi\left(\sum_{i, j} \overline{\lambda_{i}} \lambda_{j} u_{i}^{*} x u_{j}\right)\right| \leq \sum_{i, j}\left|\phi\left(\overline{\lambda_{i}} \lambda_{j} u_{i}^{*} x u_{j}\right)\right|
$$




$$
\leq \sum_{i, j}\left|\lambda_{i} \lambda_{j}\right| \phi(I)^{1 / 2} \phi\left(u_{j}^{*} x^{*} x u_{j}\right)^{1 / 2} .
$$

If $f$ is a projection, then

$$
0 \leq \psi(f) \leq \sum_{i, j}\left|\lambda_{i} \lambda_{j}\right|\|\phi\|^{1 / 2} \phi\left(u_{j}^{*} f u_{j}\right)^{1 / 2} .
$$

Since $\phi$ is singular, choose by 2.2 (ii) a projection $0 \neq e \leq f$ such that $\phi\left(u_{j}^{*} f u_{j}\right)=$ $0, j=1,2,3,4$, which shows that $\phi(f)=0$, so $\phi$ is singular.

Recall that if $N \subset M$ are von Neumann algebras, a map $\Phi: M \rightarrow B(H)$ is called $N$ - modular if $\Phi(n m)=n \Phi(m)$ and $\Phi(m n)=\Phi(m) n$ for every $m \in M$ and $n \in N$. If $\phi$ is a linear functional on $M$, then $\phi$ is $N$ - invariant if $\phi(n m)=\phi(m n)$ for all $m \in M$ and $n \in N$.

2.4. Proposition. Let $M$ be a type $\mathrm{II}_{1}$ factor with a hyperfinite subfactor $R$. If there exists a singular state on $M$ extending the trace on $R$, then there exists a singular R-invariant state on $M$.

Proof. Let $\psi$ be a singular state extension of the trace $\tau$ on $R$. Let $\left(R_{n}\right)$ be an increasing sequence of type $\mathrm{I}_{2^{n}}$ subfactors of $R$ whose union is strongly dense in $R$. Define $\psi_{n}(x)=\int \psi\left(u^{*} x u\right) d \mu_{n}(u)$ where $\mu_{n}$ is the Haar measure on the compact unitary group of $R_{n}$. Since the above integrals are point-norm limits of singular states (see 2.3), $\psi_{n}$ will be singular states, $R_{n^{-}}$invariant and extending the trace on $R$. Let $\omega$ be a free ultrafilter on the set of natural numbers $\mathbf{N}$ and define $\phi(x)=$ $\omega$-lim $\psi_{n}(x)$. Then $\phi$ is a singular state (this follows from 2.2(ii)) which extends the trace on $R$ and is invariant with respect to $C^{*}\left(\cup R_{n}\right)$. Our next goal is to show that $\phi$ is in fact $R$ - invariant.

Fix $m \in M$ and for every $r_{1}, r_{2} \in R$ define the map $\left(r_{1}, r_{2}\right) \rightarrow \phi\left(r_{2}^{*} m r_{1}\right)$. We have

$$
\begin{gathered}
\left|\phi\left(r_{2}^{*} m r_{1}\right)\right| \leq \phi\left(r_{2}^{*} r_{2}\right)^{1 / 2} \phi\left(r_{1}^{*} m^{*} m r_{1}\right)^{1 / 2} \\
\leq \phi\left(r_{2}^{*} r_{2}\right)^{1 / 2}\|m\| \phi\left(r_{1}^{*} r_{1}\right)^{1 / 2}=\|m\| .\left\|r_{2}\right\|_{2}\left\|r_{1}\right\|_{2} .
\end{gathered}
$$

This induces a bounded linear map $E: M \rightarrow B\left(L^{2}(R, \tau)\right)$ by

$$
\left(E(m) r_{1}, r_{2}\right)=\phi\left(r_{2}^{*} m r_{1}\right) .
$$

Clearly $E$ is a positive map, $\|E(m)\| \leq\|m\|$ and $E(r)=r$ for all $r \in R$. Also, for $r \in R$,

$$
\left(r E(m) r_{1}, r_{2}\right)=\left(E(m) r_{1}, r^{*} r_{2}\right)=\phi\left(r_{2}^{*} r m r_{1}\right)
$$

and

$$
\left(E(r m) r_{1}, r_{2}\right)=\phi\left(r_{2}^{*} r m r_{1}\right)
$$

which shows that $E$ is left $R$ - modular. Similarly, $E$ is right $R$-modular.

If $r_{0}$ is any operator in $C^{*}\left(\cup R_{n}\right)$ and $J$ denotes the canonical conjugation on $L^{2}(R, \tau)$, we have

$$
\left(J r_{0} J E(m) r_{1}, r_{2}\right)=\left(E(m) r_{1}, J r_{0}^{*} J r_{2}\right)=\left(E(m) r_{1}, r_{2} r_{0}\right) \phi\left(r_{0}^{*} r_{2}^{*} m r_{1}\right)
$$

and

$$
\left(E(m) J r_{0} J r_{1}, r_{2}\right)=\left(E(m) r_{1} r_{0}^{*}, r_{2}\right)=\phi\left(r_{2}^{*} m r_{1} r_{0}^{*}\right)
$$


Invariance of $\phi$ shows that $E(m)$ commutes with $J C^{*}\left(\cup R_{n}\right) J$, and since $J R J=$ $R^{\prime}$, we get $E(M) \subset R$, so $E$ is a conditional expectation. The singularity of $E$ follows easily from

$$
\tau(E(m))=(E(m) I, I)=\phi(m) .
$$

For every unitary $u \in R$

$$
\phi\left(u^{*} m u\right)=\tau\left(E\left(u^{*} m u\right)\right)=\tau\left(u^{*} E(m) u\right)=\tau(E(m))=\phi(m)
$$

which shows that $\phi$ is $R$-invariant, and the proof is complete.

We record the existence of $E$ in the following

2.5. Corollary. If there exists a singular state on $M$ which extends the trace on $R$, then there exists a singular conditional expectation from $M$ onto $R$.

\section{The RElative DiXMIER PROPERTy}

In this section we introduce the relative Dixmier property, following [2], and we establish the connection with singular states.

3.1. Definition. Let $M$ be a $\mathrm{II}_{1}$ factor. A unital subalgebra $B \subset M$ is called a Dixmier subalgebra if the norm-closed convex hull of $\left\{u^{*} m u ; u \in B\right.$ unitary $\}$ contains $\tau(m) I$ for every $m \in M$.

3.2. Remark. If $B \subset M$ is a Dixmier subalgebra then $B^{\prime} \cap M=\mathbf{C} I$. Indeed, if $p \in B^{\prime} \cap M$ is a projection, then the relative Dixmier property shows that $p=\tau(p) I$, which implies $p=0$ or $p=I$.

3.3. Proposition. Let $M$ be a $\mathrm{II}_{1}$ factor with a separable Dixmier subfactor $N$. Then there is no singular state on $M$ which extends the trace on $N$.

Proof. Let $\tau$ denote the trace on $N$ and suppose there exists a singular state $\phi$ on $M$ such that $\phi(n)=\tau(n)$ for every $n \in N$. We repeat the construction in the proof of 2.4. Fix $m \in M$ and for every $n_{1}, n_{2} \in N$ define the map $\left(n_{1}, n_{2}\right) \rightarrow \phi\left(n_{2}^{*} m n_{1}\right)$. We have

$$
\begin{gathered}
\left|\phi\left(n_{2}^{*} m n_{1}\right)\right| \leq \phi\left(n_{2}^{*} n_{2}\right)^{1 / 2} \phi\left(n_{1}^{*} m^{*} m n_{1}\right)^{1 / 2} \\
\leq \phi\left(n_{2}^{*} n_{2}\right)^{1 / 2}\|m\| \phi\left(n_{1}^{*} n_{1}\right)^{1 / 2}=\|m\| \cdot\left\|n_{2}\right\|_{2}\left\|n_{1}\right\|_{2} .
\end{gathered}
$$

This induces a bounded linear map $E: M \rightarrow B\left(L^{2}(N, \tau)\right)$ by

$$
\left(E(m) n_{1}, n_{2}\right)=\phi\left(n_{2}^{*} m n_{1}\right) .
$$

Clearly $\|E(m)\| \leq\|m\|$ and $E(n)=n$ for all $n \in N$. A computation similar to the one in 2.4 shows that $E$ is $N$ - modular.

We now show that $E$ is singular. Fix $p$ a nonzero projection in $M$ and $\left(n_{k}\right)_{k \geq 1}$ a countable subset of $\mathrm{M}$ dense in $L^{2}(N, \tau)$. Choose, by 2.2 (ii), a projection $0 \neq e \leq p$ such that $\phi\left(n_{k}^{*} e n_{k}\right)=0$ for all $k$. Then for all $k$

$$
\left(E(e) n_{k}, n_{k}\right)=\phi\left(n_{k}^{*} e n_{k}\right)=0 ;
$$

therefore $E(e)=0$. Now $E\left(u^{*} m u\right)=u^{*} E(m) u$ for every unitary $u \in N$. The relative Dixmier property implies that there are positive numbers $\lambda_{i}$ of sum 1 and unitaries $u_{i} \in N$ such that

$$
\sum_{1}^{n} \lambda_{i} u_{i}^{*} E(m) u_{i}=E\left(\sum_{1}^{n} \lambda_{i} u_{i}^{*} m u_{i}\right)
$$


is within an epsilon from $E(\tau(m) I)=\tau(m) I$. In particular, for $m=e$ we get $\tau(e)=0$, contradiction.

We will show (4.7) that one cannot remove the separability assumption on $N$.

\section{EXAMPLES AND APPLiCATiONS}

4.1. If $N \subset M$ are separable $\mathrm{II}_{1}$ factors and $\operatorname{dim} N^{\prime} \cap M=\infty$, there exist singular states on $M$ which extend the trace on $N$. To see this, let $\left(e_{n}^{\prime}\right)$ be a countable family of mutually orthogonal projections in $N^{\prime} \cap M, \sum e_{i}^{\prime}=I$. Define the unit vectors in $L^{2}(M, \tau) \xi_{n}=e_{n}^{\prime} \tau\left(e_{n}^{\prime}\right)^{-1 / 2}$. Clearly $\xi_{n}=e_{n}^{\prime} \xi_{n}$ and define on $M \phi_{n}(x)=\left(x \xi_{n}, \xi_{n}\right)$. Then $\phi_{n}$ are normal states on $M$ and for $x \in N$ we have

$$
\phi_{n}(x)=\left(x \xi_{n}, \xi_{n}\right)=\tau\left(x \xi_{n} \xi_{n}^{*}\right)=\tau\left(x e_{n}^{\prime} / \tau\left(e_{n}^{\prime}\right)\right)=\tau(x) .
$$

The last equality is $\tau\left(x e_{n}^{\prime}\right)=\tau(x) \tau\left(e_{n}^{\prime}\right)$ and it follows from the uniqueness of the trace on $N$. Now let $\omega$ be a free ultrafilter on $\mathbf{N}$ and define $\phi(x)=\omega$-lim $\phi_{n}(x)$. It follows that $\phi$ is a state on $M$ which extends the trace on $N$. Also, for every $k \geq 1, \phi\left(I-e_{k}^{\prime}\right)=0$ and 2.2(ii) implies that $\phi$ is singular.

4.2. S. Popa proved in [6] that if $M$ and $N$ are $\mathrm{II}_{1}$ factors, then $M$ has trivial relative commutant in $M * N$. (In fact [6], 4.1, is a more general result. See also $[1], 2.5$.$) For the basics on free product factors we refer to [9]$.

For any projections $e \in M$ and $f \in N$ the definition of freeness shows that

$$
\tau((e-\tau(e) I)(f-\tau(f) I))=0
$$

which implies that $\tau(e f)=\tau(e) \tau(f)$.

Choose now an arbitrary decreasing sequence of projections $\left(f_{n}\right)$ in $N$ with so-lim $f_{n}=0$ and define on $M * N$ the states

$$
\phi_{n}(x)=\tau\left(x f_{n}\right) / \tau\left(f_{n}\right)
$$

For any projection $e \in M$ we have $\phi_{n}(e)=\tau\left(e f_{n}\right) / \tau\left(f_{n}\right)=\tau(e)$ so $\phi_{n}$ are extensions of the trace on $M$. If $\omega$ is a free ultrafilter on $\mathbf{N}$, define $\phi(x)=\omega$-lim $\phi_{n}(x)$. Since $\phi\left(I-f_{n}\right)=0$ for all $n, \phi$ is a singular state on $M * N$ which extends the trace on $M$.

4.3. It now follows from 3.3 that no separable $M$ can be a Dixmier subfactor of $M * N$. If $M=R$, the hyperfinite type $\mathrm{II}_{1}$ factor, then 2.4 and 2.5 show that there exist $R$ - invariant singular states on $R * N$, hence singular conditional expectations from $R * N$ onto $R$.

In view of 2.4 and 3.3 we ask the following

4.4. Question. Does every separable $\mathrm{II}_{1}$ factor $M$ have a Dixmier subfactor ? Can this Dixmier subfactor be hyperfinite?

4.5. Yet another situation where the above ideas apply is the commutative case, which we only present for comparison. If $A=B=L^{\infty}([0,1])$, the constructions in 4.1 and 4.2 can be repeated almost word by word to exhibit a singular state on $A \bar{\otimes} B$ (i.e. a measure on $[0,1] \times[0,1]$ singular with respect to the Lebesgue product measure) which extends the (normal) trace (i.e. the Lebesgue measure) on $A$.

4.6. Let $M$ be a separable $\mathrm{II}_{1}$ factor and $N$ be a separable subfactor of $M^{\omega}$. By a theorem of Popa [7] there is a separable diffuse abelian algebra $A \subset M^{\omega}$ which is free with respect with $N$. The construction in 4.2 applies to a decreasing sequence of projections in $A$ to obtain a singular state on $M^{\omega}$ which extends the trace on 
$N$. Following 3.3, no separable $N \subset M^{\omega}$ is a Dixmier subfactor. Recall, however, ([7]) that if $Q \subset M$ is an irreducible subfactor then $Q^{\omega} \subset M^{\omega}$ is a (nonseparable) Dixmier subfactor.

4.7. Since $M$ has trivial relative commutant in $M * N([6])$, it follows that $M^{\omega}$ is a Dixmier subfactor of $(M * N)^{\omega}([7])$. We will exhibit a singular state on the latter which extends the trace on the former, thus showing that the separability assumption in 3.3 cannot be dropped. Using the notation in 4.2 and 4.3 , if $f_{n}$ is a sequence of projections in $N$ decreasing to 0 , define the sequence $F_{n}=\left(f_{n}, f_{n}, \ldots\right)$ of projections in $(M * N)^{\omega}$, decreasing to 0 . If $E$ is any projection in $M^{\omega}$, there are projections $e_{n}$ in $M$ such that $E=\left(e_{1}, e_{2}, \ldots\right)$. Since $\tau\left(e_{i} f_{n}\right)=\tau\left(e_{i}\right) \tau\left(f_{n}\right)$, we get $\tau\left(E F_{n}\right)=\tau(E) \tau\left(F_{n}\right)$, so the construction in 4.2 applies to obtain a singular state on $(M * N)^{\omega}$ which extends the trace on $M^{\omega}$.

\section{ACKNOWLEDGEMENTS}

I would like to thank Professors Liming Ge, David Pitts, Sorin Popa and Roger Smith for useful discussions.

\section{REFERENCES}

1. L. Ge, On maximal injective subalgebras of factors, Adv. Math. 118 (1996), 34-70.

2. H. Halpern, V. Kaftal and G.Weiss, The relative Dixmier property in discrete crossed products, J. Funct. Anal. 69 (1986), 121-140. MR 87k:46137

3. R. V. Kadison and I. M. Singer, Extensions of pure states, Amer. J. Math. 81 (1959), 383-400. MR 23:A1243

4. R. V. Kadison and J. R. Ringrose, Fundamentals of the theory of operator algebras, vol. II, Academic Press, New York, 1986. MR 88d:46106

5. S. Popa, On a problem of R. V. Kadison on maximal abelian *-subalgebras in factors, Invent. Math. 65 (1981), 269-281. MR 83g:46056

6. - Markov traces on universal Jones algebras and subfactors of finite index, Invent. Math. 111 (1993), 375-405. MR 94c:46128

7. _ Free-independent sequences in type $\mathbf{I I}_{1}$ factors and related problems, Astérisque, $\mathbf{2 3 2}$ (1995), 187-202. MR 97b:46080

8. M. Takesaki, On the singularity of a positive linear functional on operator algebras, Proc. Jap. Acad. 35 (1959), 365-366. MR 22:3991

9. D. V. Voiculescu, K. J. Dykema and A. Nica, Free random variables, CRM Monograph Series, vol.1, AMS, Providence RI, 1992. MR 94c:46133

Department of Mathematics and Statistics, University of Nebraska, Lincoln, NeBRASKA 68588

Current address: Division of Mathematical Science, 241 Schaeffer Hall, University of Iowa, Iowa City, Iowa 52242

E-mail address: fpop@stat.uiowa.edu 